\title{
Can We Do Even Better? Preoperative Imaging Overestimates the Tumor Size in Pancreatic Neuroendocrine Neoplasms Associated with Multiple Endocrine Neoplasia Type 1
}

\author{
Chad G. Ball ${ }^{1}$
}

Published online: 15 November 2017

(C) Société Internationale de Chirurgie 2017

Dr. Polenta and her colleagues have provided our neuroendocrine surgical community with a very interesting and detailed assessment of the accuracy of preoperative imaging for pancreatic neuroendocrine neoplasms (pNEN) in patients with multiple endocrine neoplasia type 1 (MEN 1) [1]. As we know, size criteria for these tumors often act as triggers for major surgical interventions and as a result, a significant risk of postoperative morbidity and mortality.

The authorship concluded that although magnetic resonance imaging (MRI) and computed tomography (CT) performed reasonably well with regard to radiologic versus pathologic dimensions of these lesions, endoscopic ultrasound (EUS) frequently overestimated these neoplasms. Given the extensive user dependency associated with ultrasonography of all forms, this finding is not overly surprising. It is, however, an extremely important confirmation within our field. It will not only impact which imaging modality we choose to evaluate and potentially surveil our patients with pNEN, but may also modify the individual patients we choose to resect. As with most surgical imaging modality data, these findings are significant food for thought.

Dr. Polenta's team evaluated 44 patients in this singlecenter series to provide us with their detailed conclusions. Although small single-center series have provided us with some incredibly important signals for further study and evaluation within the literature in general, 'bigger data' is becoming increasingly important to recognize. More specifically, as a surgical community struggling to improve the care of patients with less common (and sometimes quite rare) diagnoses, we must continue to engage in multicenter collaboration, discussion and publication. Some subspecialty communities (trauma and critical care for example) have displayed improved success in engaging this model (multicenter retrospective collaborations) when compared to others. Anecdotally, we are also obtaining the increased and exciting sense that young surgeons appear to be developing these pathways to collaboration at an increased rate than those of us who preceded them. While prospective randomized trials continue to represent our gold standard in eliminating bias and providing strong evidence, much can still be gleaned and learned from retrospective collaborative series. Clearly some topics lend themselves to this approach in a much preferred manner than others, but we must continue to strive to work together, share our individual experiences and publish our collective analyses in a thought-provoking fashion that moves our small and large fields alike forward.

\section{Reference}

1. Polenta V, Slater EP, Kann PH et al (2017) Preoperative imaging overestimates the tumor size in pancreatic neuroendocrine neoplasms associated with multiple endocrine neoplasia type 1 . World J Surg. https://doi.org/10.1007/s00268-017-4317-8
Chad G. Ball

ball.chad@gmail.com

University of Calgary, Surgery, Calgary, Canada 